\title{
3.10 RESULTS OF A SEARCH FOR VISIBLE PULSARS
}

\author{
P. HOROWITZ \\ Harvard University \\ C. PAPALIOLIOS and N. P. CARLETON \\ Smithsonian Astrophysical Observatory and Harvard University
}

\begin{abstract}
A number of interesting celestial objects, including X-ray sources, nova and supernova remnants, white dwarfs, infrared stars, planetary nebulae, radio sources, and some other peculiar nebulosities have been searched with Fourier and correlation techniques for the presence of optical pulsars. The sensitivity of these methods was established with synthetic data and by observations of the Crab Nebula pulsar, the minimum detectable signal (5 standard deviations above noise) being approximately 20th visual magnitude, time averaged. To this limit no new pulsars were found over the range of periods searched from $5 \mathrm{msec}$ to several seconds.
\end{abstract}

For the past two years we have been attempting to locate visible pulsars, both at known radiofrequency pulsar sites and elsewhere, using Harvard's 61-inch telescope at Agassiz Station. The search techniques are completely straightforward, consisting of (a) multichannel scaling of the light intensity modulo the pulsar period, for known radiofrequency pulsars, and (b) performing a discrete Fourier transform on the sampled light intensity for other candidates where the period is not known a priori. There are many very interesting details connected with these techniques, but since they will appear shortly in a formal journal publication we will confine ourselves here to some of the motivation for the search, and the results.

Besides the known pulsars, we examined the objects listed in Table I, the most interesting of which are the supernova remnants. The Crab and Vela pulsars establish a connection between pulsars and supernova remnants, strengthened by the rough agreement of ages deduced by quite separate means. One therefore expects to find pulsars at the sites of galactic supernovae, and, furthermore, recent supernovae are likely to contain faster (and possibly visible) pulsars. These expectations are based largely upon the characteristics of the Crab pulsar - the youngest known pulsar and the only one visible at optical wavelengths, but simple theoretical arguments lead to the same conclusions. It is not difficult to estimate the peak energy of photons emitted through synchrotron radiation for several popular pulsar theories. In the 'near field' models one gets (Horowitz, 1970)

$$
U_{\text {peak }} \text { (photons) } \approx 3 \times 10^{7} \Omega^{3 / 2} B_{12}^{3 / 4} \mathrm{eV}
$$

and in the 'light cylinder' theories

$$
U_{\text {peak }}(\text { photons }) \approx 4 \times 10^{6} \Omega^{7 / 4} B_{12}^{3 / 4} \mathrm{eV}
$$

where $\Omega$ is the neutron-star's angular frequency, $(\Omega \sim 10 \mathrm{rad} / \mathrm{sec})$ and $B_{12}$ is the surface field in units of $10^{12}$ gauss. These are surprisingly high energies, and seem to 
TABLE I

Objects examined for visible pulses




indicate that optical synchrotron radiation is possible for most pulsars. We have not said anything about intensity, though, and that depends upon factors like the rate of injection of particles into the system, a process that could depend strongly upon $\Omega$.

In order to learn how faint a pulsar could be detected with transform techniques, we made optical observations of the Crab pulsar. The Fourier transform of two minutes of data clearly shows the fundamental frequency and seven harmonics, with amplitudes about 30 standard deviations above the noise. By transforming longer strings of data, and summing the amplitudes of harmonically related frequencies. the pulsar shows itself as peaks more than 100 standard deviations above noise, implying that a similar pulsar $3^{\mathrm{m}} 5$ fainter would be detected with this technique. From such observations, as well as from computer experiments, we have established the sensitivity of the search techniques, and the estimated limiting magnitudes for each object examined is listed in the table. The range of frequencies searched goes from the inverse of the observation time up to half the sampling frequency, though seeing fluctuations frequently reduced the sensitivity to signals with periods greater than a few seconds. In most cases periods from $5 \mathrm{msec}$ to about 10 seconds were within the range of the search.

The remainder of Table I is mostly self-explanatory. The central stars of planetary nebulae, nova remnants, white dwarfs, and some of the "other objects" are stellar, and were easy to examine. They were chosen particularly for unusual characteristics, e.g., lack of any spectral lines (the white dwarfs) or suspected variability (NGC 7662). In the planetary nebula NGC 7027, no central star can be seen, but searches with a 4.6" diameter aperture near the brightest region failed to disclose any pulsation. The X-ray sources SCO-X1 and CYG-XR2 were sampled at $0.1 \mathrm{msec}$ intervals, in addition to the usual $2.5 \mathrm{msec}$ rate, in order not to overlook a very short-period pulsation, that has been suggested (Tucker, 1969).

The supernova remnants, certainly the most interesting candidates, posed a special problem of large angular size and absence of striking visible features (as in the Crab). Faint nebulosity has been discovered near the position of Kepler's and Tycho's supernovae, and both Tycho's supernova and Cas A show a characteristic circular structure, a few minutes in diameter, at radiofrequencies. Cas A also has some nebulosity, is an X-ray source, and is widely believed to be a supernova remnant. In these cases, as well as the suspected remnants NGC 6888, S 22, and 3C58, many stars in the vicinity were searched. An extragalactic supernova was observed at a time close to its peak brightness (in NGC 3556), as well as the position of the widely observed supernova event in the Andromeda galaxy in 1885. The YT objects are peculiar nebulosities suggested by Dr. Y. Terzian of Cornell. The list even includes a quasar (3C273) and an infrared star, the 'Taurus Source' (Neugebauer et al., 1965). Three radiofrequency pulsars were observed with Dr. Frank Low (University of Arizona) at $5 \mu-20 \mu$, using his helium-cooled germanium bolometer attached to the 28 " reflector at Mt. Lemmon.

With the single exception of the Crab pulsar, NP 0531, no optical pulsar-like fluctuations were observed in any of the objects searched. Since observations of the Crab 
pulsar confirmed that the technique was adequate to detect similar pulsars at least 3 magnitudes fainter, a number of conclusions are possible:

(1) Not all supernova events produce neutron stars.

(2) Not all neutron stars are pulsars.

(3) Not all pulsars produce optical pulses.

(4) Not all optically pulsing pulsars are visible from an arbitrary direction.

(5) Other optical pulsars are outside the regions of our search (in period, brightness, region of sky, etc.).

It is difficult to choose among these speculative propositions. It is worthwhile noting, however, that the uniqueness of the Crab Nebula among recent supernova remnants, particularly in its X-ray and visible activity, suggests that (1) or (5) may be true. The failure to observe any visible objects at the sites of radiofrequency pulsars, albeit old ones gives support to (3). And the polarization properties of the one visible pulsar (Kristian et al., 1970) strongly hint at (4).

We would like to make two final remarks. One is that the Crab Nebula, when viewed at radiofrequencies, is brightest at the center, whereas other supernova remnants appear as hollow shell sources. This property, as well as some of the other remarkable features of the Crab (X-rays, optical synchrotron radiation, compact radiofrequency source, etc.), is presumably due to the continued excitation by the remnant pulsar, and suggest that these other supernova remnants have no associated pulsars. Of the objects studied, only $3 \mathrm{C} 58$ and $3 \mathrm{C} 386$ have similar radio structure to the Crab, but $3 \mathrm{C} 58$ has no visible counterpart on Palomar Sky Survey plates, and neither source has the other peculiar characteristics of the Crab. Here, too, we found no pulsar.

The other remark concerns Prentice's (1970) suggestion that most pulsars may be runaway stars, having motion relative to the original gaseous remnant. He assigns a nearby nebula to each of a number of radiofrequency pulsars, and suggests that only young pulsars might still be found within their nebular remnants (Crab, Vela). Perhaps the remnant star (if any) has already moved appreciably from the center of the nebulosity seen in the supernova remnants examined and was thereby overlooked.

As for the other objects looked at (nova remnants, planetaries, etc.), if one believes that they are what they seem to be, and that current descriptions are accurate, then there is little reason to expect them to pulsate at these frequencies (though whitedwarf radial pulsations might well be expected, and have been looked for previously (Lawrence et al., 1967)). However, the techniques developed in the course of this search are capable of detecting pulsations many orders of magnitude faster than conventional astronomical photometry, and that alone seems to justify some general looking around at familiar objects.

\section{References}

Horowitz, P.: 1970, 'Optical Studies of Pulsars', Thesis, Harvard University.

Kristian, J. and Visvanathan, N., Westphal, J. A., and Snellen, G. H.: 1970, Astrophys. J. 162, 475. Lawrence, G. M., Ostriker, J. P., and Hesser, J. E.: 1967, Astrophys. J. 148, L162. 
Neugebauer, G., Martz, D. E., and Leighton, R. B.: 1965, Astrophys. J. 142, 399.

Prentice, A. J. R.: 1970, Nature 225, 438.

Tucker, W.: 1969, Nature 223, 1250.

\section{Discussion}

J.P. Ostriker: It seems that in some cases your limits are high enough to allow a substantial $(10 \%-50 \%)$ periodic component in the objects investigated.

P. Horowitz: All values given are upper limits, since no pulsations were seen, except of course in the Crab (which we used as a calibrating source). The limits vary since they are a function of observation time, aperture, brightness of the object etc.

$J$. A. Roberts: Because the pulse shapes clearly have a very large harmonic content I would not expect a Fourier transform method to be very sensitive method of searching for pulsations. A technique, such as superposed epoch, which does not spread the signal into many places in the transform plane, would seem more suitable.

P. Horowitz: I agree that the Fourier Transform is not best suited to searching for pulsations of short duty cycle, but if you add the amplitudes of harmonically related components then the sensitivity is only the fourth root of the duty cycle poorer than the optimum method, i.e. 'stacking' modulo the correct period. The Staelin Fast Folding Algorithm could be used to accomplish the latter, but the Fast Fourier Transform is so much faster, when searching a wide range of frequencies, that we preferred to use it instead; in all our searches we summed harmonically related frequencies and examined the resulting array. 